\title{
Evaluation of Flavonoids and Furanocoumarins from Citrus bergamia (Bergamot) Juice and Identification of New Compounds
}

\author{
Claudio Gardana ${ }^{1, *}$, Federico Nalin ${ }^{2}$ and Paolo Simonetti ${ }^{1}$
}

1 DiSTAM - Department of Food Science and Microbiology, Division of Human Nutrition, University of Milan, 20133 Milan, Italy; E-mail: paolo.simonetti@unimi.it (Paolo Simonetti)

2 Specchiasol s.r.l., Via Rizzi 1/3 - 37012 Bussolengo (Vr), Italy;

E-mail: federico.nalin@specchiasol.it (Federico Nalin)

* Author to whom correspondence should be addressed; E-mail: claudio.gardana@unimi.it; Tel.: +3902 50316072; Fax +390250316071.

Received: 1 August 2008; in revised form: 1 September 2008 / Accepted: 9 September 2008 / Published: 18 September 2008

\begin{abstract}
Bergamot juice (BJ) contains different classes of flavonoids (e.g. flavanones and flavones) that can exert beneficial effects on human health. The aim of this study was to evaluate the qualitative and quantitative composition of a BJ obtained from fruits harvested in Southern Italy (Calabria) at the end of their maturation period. The identity of several flavonoids and furanocoumarins was assessed by co-chromatography, UV spectra and molecular weight comparison. The unknown compounds were dissociated by induced collision (CID-MS) and their identity established through the characteristic ions product. By this approach a complete profile of about twenty compounds (furano-coumarins, flavonoids C- and O-glycosides) present in BJ was obtained. Furthermore, three acylated flavanones, present in amounts of $20.1 \pm 1.1,89.3 \pm 2.2$ and $190.1 \pm 3.1 \mathrm{mg} / \mathrm{L}$, respectively, and which seem to correspond to di-oxalate derivatives of neoeriocitrin, naringin and neohesperidin, were identified for the first time in BJ. The other main flavanones were naringin, neohesperidin and neoeriocitrin, and their content was 167.5 $\pm 1.8,123.9 \pm 1.7$ and $73.3 \pm 1.6 \mathrm{mg} / \mathrm{L}$, respectively. Concerning flavones, the total amount in BJ was about 160 $\mathrm{mg} / \mathrm{L}$ and the main ones were vicenin-2, stellarin-2, rhoifolin and neodiosmin. Bergapten
\end{abstract}


and bergamottin were the primary furanocoumarins in $\mathrm{BJ}$ and their amounts were $9.0 \pm 0.4$ and $18.2 \pm 0.5 \mathrm{mg} / \mathrm{L}$, respectively.

Keywords: Flavonoids; Furanocoumarins; Bergamot juice; LC; Mass spectrometry

\section{Introduction}

Flavanones represent a small group of compounds present in high concentrations in different citrus like orange, grapefruit and lemon and in lesser amounts also in tomatoes, some aromatic plants and propolis. In citrus juice the main aglycones are naringenin, hesperetin and eriodictyol glycosylated with neohesperidose, which imparts a bitter taste, or rutinose that is flavourless. Bergamot (Citrus bergamia Risso) fruit and juice (BJ) are a source of bitter taste compounds containing mainly the flavanones neohesperidin, naringin, neoeriocitrin and lesser amounts of flavones and furanocoumarins. After ingestion as food the flavanone glycosides are metabolised by human intestinal bacterial microflora to the respective aglycones [1,2], which seem to possess antioxidant [3], anticarcinogenic [4], hepatolipidemic [5] and anti-inflammatory [6] activities. In addition, naringenin and hesperetin can bind to estrogen receptors [7], or inhibit the activity of aromatase, the rate-limiting enzyme in the conversion of androgens to estrogens [8]. Naringin was found to lower total cholesterol and lowdensity lipoprotein cholesterol levels in plasma [9], and hesperidin to also significantly lower plasma triglyceride levels [10]. Chronic administration of BJ caused in rats a significant reduction in serum cholesterol, triglycerides, LDL and an increase in HDL levels [11]. Moreover, the authors observed a protective effect on the hepatic parenchyma. Bergamot juice contains diverse compounds with similar chemical structure thus a sophisticated high resolution technique is necessary for their identification and quantification. For this reason liquid chromatography coupled to DAD and mass spectrometry is the best choice for the analytical characterization of citrus [12-15]. The aim of this study was (1) to develop a LC-DAD-ESI-MS(MS) method allowing the qualitative and quantitative evaluation of flavonoids and furanocoumarins and (2) to identify unknown polyphenol compounds in the bergamot juice by LC-MS ${ }^{2}$.

\section{Results and Discussion}

The analyzed BJ contained different classes of compounds like flavonoids, cinnamic acid derivatives and furanocoumarins, whose structures are given in Figure 1. Bergamot juice is a complex food matrix and the identity of its components can be correctly assessed by LC coupled with DAD and a tandem mass spectrometer. A good separation of flavonoids in BJ was achieved with a Luna(2) $\mathrm{C}_{18}$ narrow bore column with gradient elution. Figure 2 shows the chromatograms of a BJ sample at 282 $\mathrm{nm}$ (A) and $330 \mathrm{~nm}$ (B). The identity of peaks 2, 3, 5, E, I, M, a and b was established by cochromatography, "on-line" UV spectra comparison and molecular ion evaluation. Compounds A-D showed an UV spectrum suggesting a structure of a flavone-derivative and in $\mathrm{MS}^{2}$ they yielded typical ions product corresponding to (M-H) ${ }^{-},[(\mathrm{M}-\mathrm{H})-18]^{-},[(\mathrm{M}-\mathrm{H})-90]^{-},[(\mathrm{M}-\mathrm{H})-120]^{-}$and $[(\mathrm{M}-\mathrm{H})-210]^{-}$. The losses in the MS/MS coincided with those reported by Ferreres et al. [16] for di-C-symmetric hexosyl 
flavones, suggesting that peaks A-D were 6,8-di-C-glucosyl derivatives of luteolin, apigenin, chrysoeriol and diosmetin, respectively.

Figure 1. Structures of flavonoids, cinnamic acid derivatives and furanocoumarins founds in BJ.

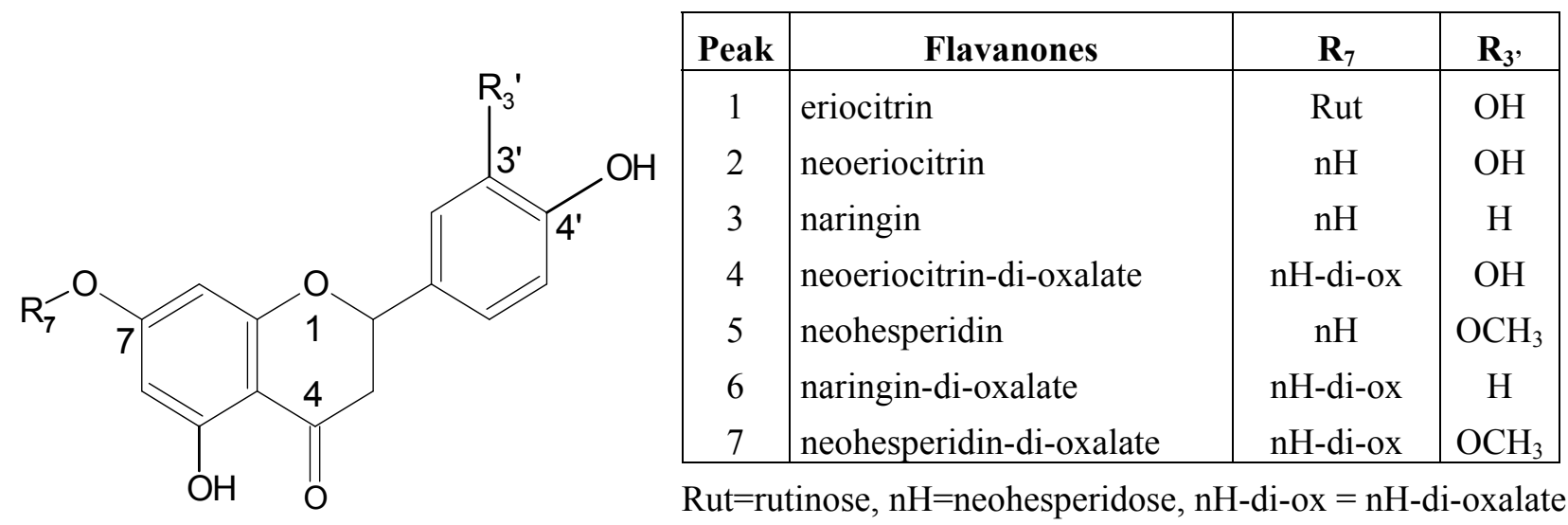

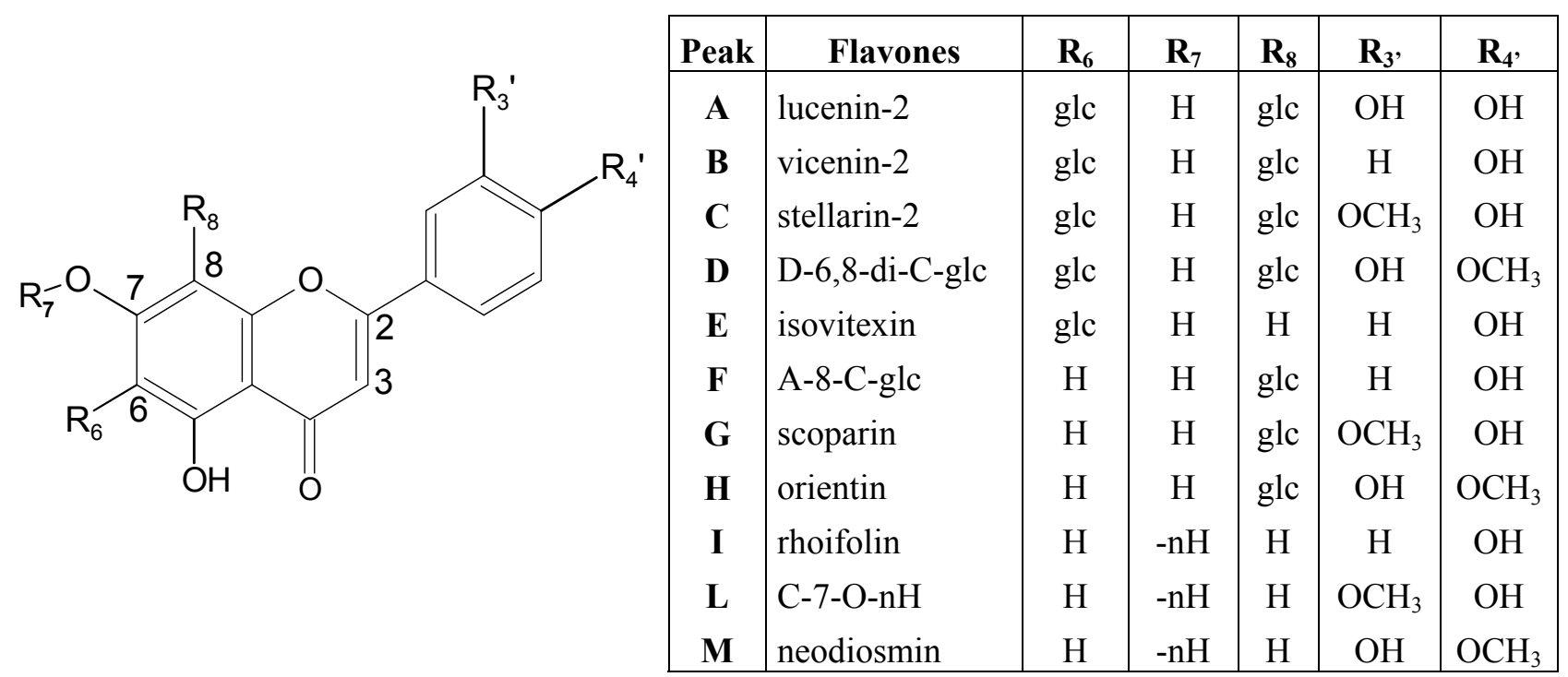

C: chrysoeriol, D: diosmetin, A: apigenin, glc: glucose<smiles>[R]c1c2ccoc2cc2oc(=O)ccc12</smiles>

Furanocoumarins

\begin{tabular}{|c|l|c|}
\hline Peak & \multicolumn{1}{|c|}{ Name } & $\mathrm{R}$ \\
\hline a & Bergapten & $\mathrm{OCH}_{3}$ \\
b & Bergamottin & $\mathrm{CH}_{2} \mathrm{CHC}\left(\mathrm{CH}_{3}\right) \mathrm{CH}_{2} \mathrm{C}\left(\mathrm{CH}_{3}\right)_{2}$ \\
\hline
\end{tabular}<smiles>[R6]OC(=O)/C=C/c1cc([R2])c(O)c(O)c1</smiles>

Caffeic acid derivatives

\begin{tabular}{|c|l|c|c|c|}
\hline Peak & \multicolumn{1}{|c|}{ Name } & $\mathrm{R}_{1}$ & $\mathrm{R}_{2}$ & \multicolumn{1}{|c|}{$\mathrm{R}_{3}$} \\
\hline X1 & di-acetyl Caffeic acid & $\mathrm{H}$ & $\mathrm{COCH}_{3}$ & $\mathrm{COCH}_{3}$ \\
X2 & Ferulic acid hexose & $\mathrm{H}$ & $\mathrm{CH}_{3}$ & hexose \\
X3 & Sinapic acid hexose & $\mathrm{OCH}_{3}$ & $\mathrm{CH}_{3}$ & hexose \\
\hline
\end{tabular}


Figure 2. Typical chromatograms of bergamot juice at $282 \mathrm{~nm}(\mathrm{~A})$ and $330 \mathrm{~nm}(\mathrm{~B})$.
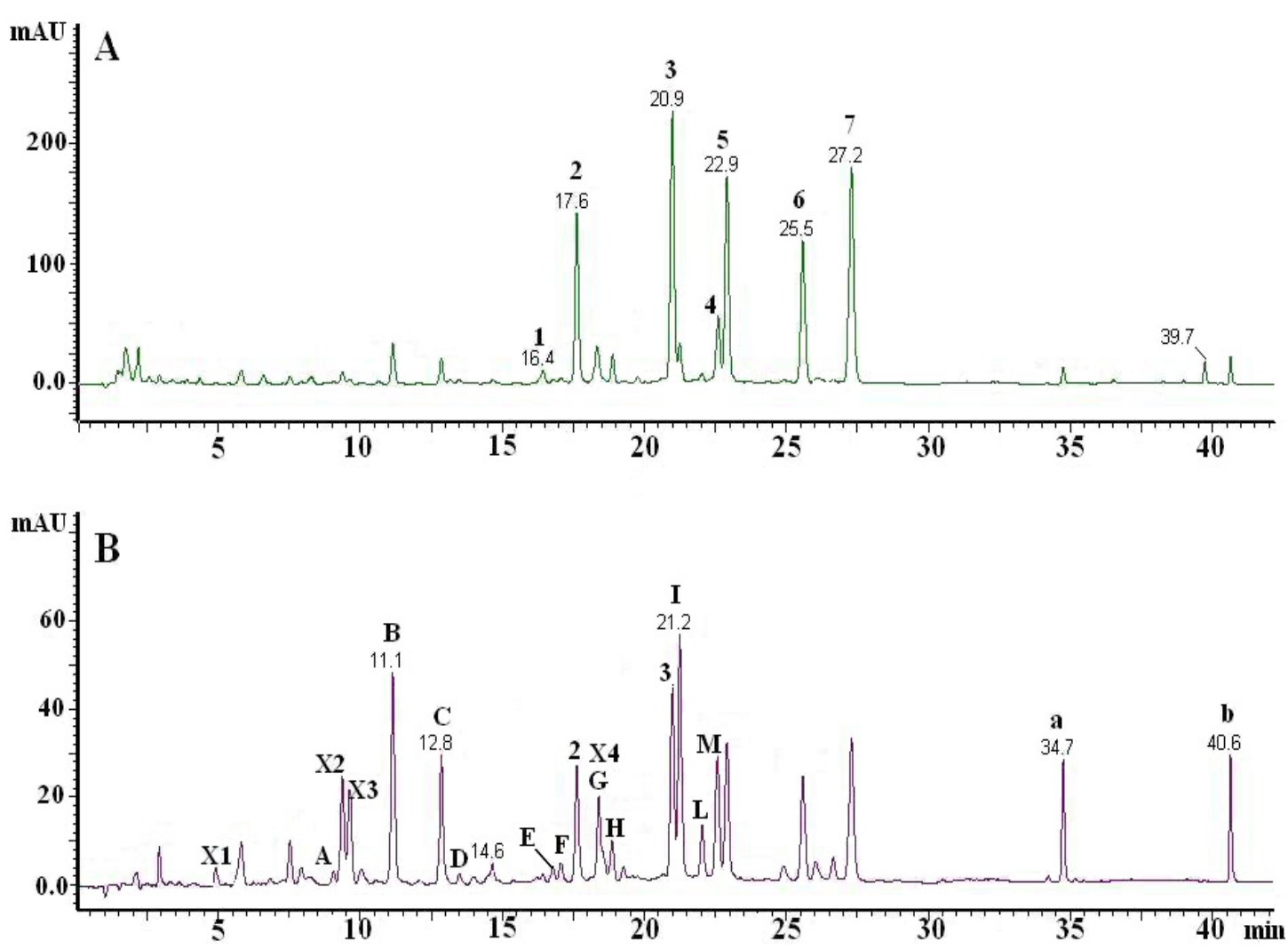

The MS/MS spectra of peaks F-H showed the presence of ions corresponding to (M-H)-, [(M-H)90] $]^{-}$and $[(\mathrm{M}-\mathrm{H})-120]^{-}$and the absence of the [(M-H)-18] $]^{-}$suggesting the presence of a single hexose moiety at the 8 position. Therefore, peaks F-H could be identified as 8-C-glucosyl derivatives of apigenin, chrysoeriol and diosmetin, respectively. Peak 1 showed an UV spectrum suggesting a structure of a flavanone-derivative and mass spectra yielded the same pseudomolecular ion with (m/z) $595 \mathrm{Da}$. At low collision energy values this ion gave an ion product with $(\mathrm{m} / \mathrm{z})^{-} 285$ Da, likely corresponding to the aglycone moiety. The mass data, combined with the UV and chromatographic behavior suggest that peak 1 was eriocitrin. Peaks 4, 6 and 7 showed an UV spectrum suggesting a flavanone-derivative structure and their mass spectra showed pseudomolecular ions with $(\mathrm{m} / \mathrm{z})^{-} 739$ $\mathrm{Da}, 723 \mathrm{Da}$ and $753 \mathrm{Da}$, respectively. The MS-MS spectra of these parent ions displayed the presence of ions corresponding to [(M-H)-62 $]^{-},[(\mathrm{M}-\mathrm{H})-102]^{-},[(\mathrm{M}-\mathrm{H})-144]^{-}$and $(\mathrm{M}-\mathrm{H})^{-}$. In positive mode peaks 4, 6 and 7 gave the same ions corresponding to $[(\mathrm{M}+\mathrm{H})-146]^{+}$and typical fragments with $(\mathrm{m} / \mathrm{z})^{+} 289$ $\mathrm{Da}, 273 \mathrm{Da}$ and $303 \mathrm{Da}$ corresponding to eriodyctiol, naringenin and hesperetin, respectively. Moreover, the CID-MS/MS of the peaks 4, 6 and 7 gave ions with $(\mathrm{m} / \mathrm{z})^{-} 151 \mathrm{Da}$, which is a common fragment obtained from flavanone aglycones not substituted on the A ring. The retention time, UV behavior, negative and positive MS-MS spectra and the presence of neohesperidosides in BJ lead to assume that peak 4, 6 and 7 could be neoeriocitrin, naringin and hesperidin esterified with two oxalic acid moieties, respectively. Figure 3 shows the fragmentation pattern of peak 7 . The presence of the ions product with $(\mathrm{m} / \mathrm{z})^{-} 609 \mathrm{Da}$, corresponding to hesperidin, lead us to suppose that ions with $(\mathrm{m} / \mathrm{z})^{-}$ $691 \mathrm{Da}$ and $651 \mathrm{Da}$ derive from the cleavage of oxalate moieties. 
Figure 3. Fragmentation pattern of the peak 7 (MW 754) obtained at lower collision energy. The ions product with $(\mathrm{m} / \mathrm{z})^{-} 609 \mathrm{Da}$ and $(\mathrm{m} / \mathrm{z})^{-} 301 \mathrm{Da}$ correspond to hesperidin and hesperetin, respectively.

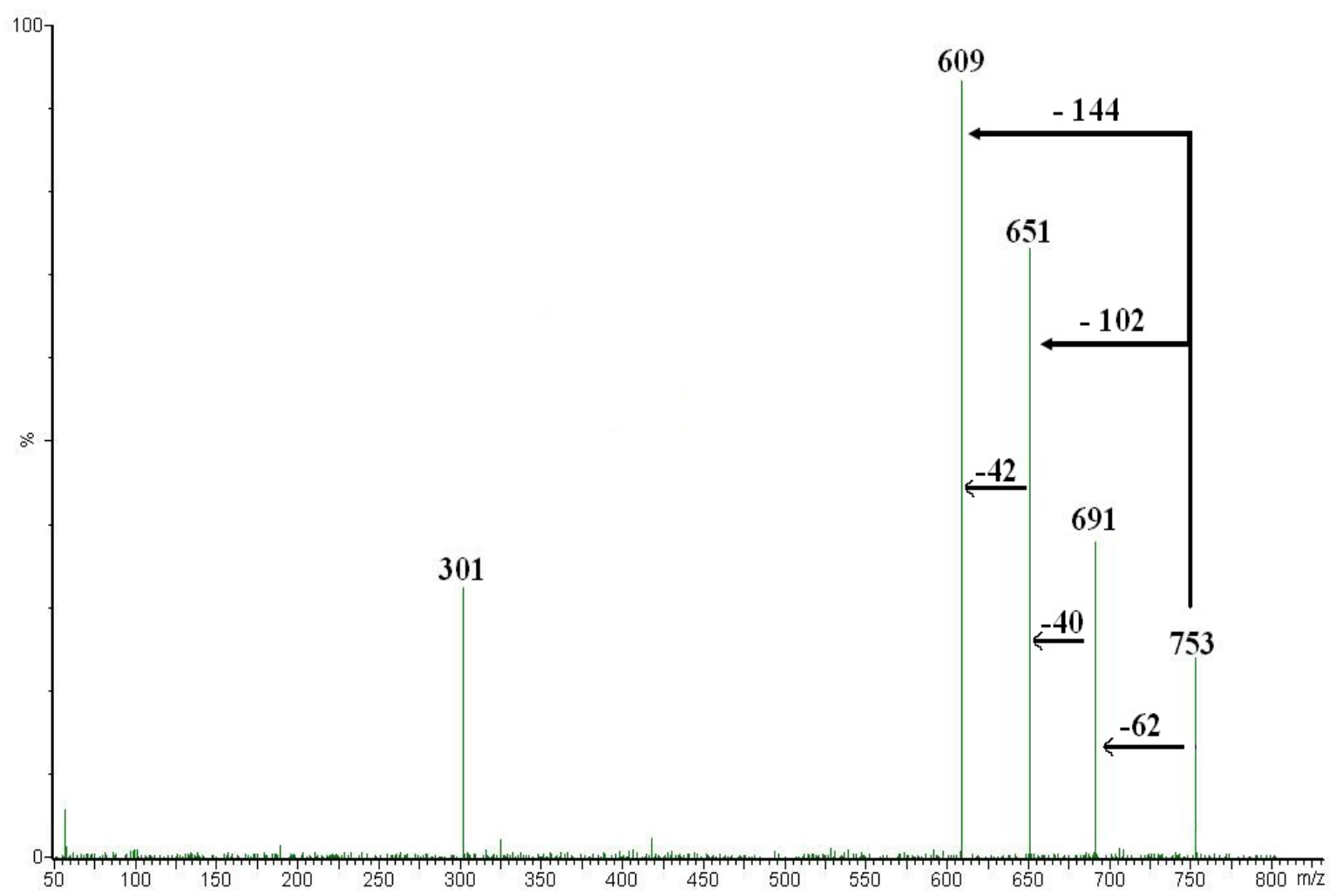

Peaks X1, X2 and X3 showed an UV spectrum suggesting to the structure of 3,4-di-hydroxylatedcinnamic acid derivatives and displayed $[\mathrm{M}-\mathrm{H}]^{-}$ions at $(\mathrm{m} / \mathrm{z})^{-} 263 \mathrm{Da}, 355 \mathrm{Da}$ and $385 \mathrm{Da}$, respectively. Peak X1 showed ions with (m/z)-221 Da [(M-H)-42]', (m/z)-179 Da (caffeic acid) [(M$\mathrm{H}])-84]^{-}$and $[\mathrm{m} / \mathrm{z}]^{-} 161 \mathrm{Da}$ (caffeic acid- $\mathrm{H}_{2} \mathrm{O}$ ) in its $\mathrm{MS}^{2}$ spectra. Based on the above observations peak X1 was tentatively identified as caffeic acid esterified with two acetic acid moieties.

In the $\mathrm{MS}^{2}$ spectra of peak X2 the main fragments were the ions with $(\mathrm{m} / \mathrm{z})^{-} 193 \mathrm{Da}$ (ferulic acid) [(M-H)-162] $]^{-},(\mathrm{m} / \mathrm{z})^{-} 175$ [ferulic acid- $\left.\mathrm{H}_{2} \mathrm{O}\right]^{-}$and $[\mathrm{m} / \mathrm{z}]^{-} 163 \mathrm{Da}\left[\left(\text { ferulic acid- } \mathrm{OCH}_{3}\right)\right]^{-}$. Analogously, compound X3 showed losses from the pseudomolecular ion [M-H] $]^{-}$of $162 \mathrm{Da}$ (hexosyl moiety) to give typical ions with $(\mathrm{m} / \mathrm{z})^{-} 223 \mathrm{Da}$ (sinapic acid). In the $\mathrm{MS}^{2}$ spectra of peak $\mathbf{X 3}$ ions with $(\mathrm{m} / \mathrm{z})^{-} 205 \mathrm{Da}$ (sinapic acid- $\left.\mathrm{H}_{2} \mathrm{O}\right)$ and $(\mathrm{m} / \mathrm{z})^{-} 163 \mathrm{Da}\left[\left(\text { sinapic acid- } \mathrm{OCH}_{3}-\mathrm{OCH}_{3}\right)\right]^{-}$were also present. Thus, it seems that peaks X2 and $\mathbf{X 3}$ were ferulic and sinapic acid esterified both with one hexosyl moiety, reasonably glucose.

Peak X4 yielded a UV spectrum with maxima at $250 \mathrm{~nm}$ and a shoulder at $275 \mathrm{~nm}$, and pseudomolecular ions with $(\mathrm{m} / \mathrm{z})^{-} 397 \mathrm{Da}$. The $\mathrm{MS}^{2}$ analysis of this precursor gave ions with $(\mathrm{m} / \mathrm{z})^{-}$ $235 \mathrm{Da}$ [(M-H)-162]', which could correspond to the aglycone moiety. Thus, Peak X4 could be considered the hexosyl derivative of an aglycone with molecular weight $236 \mathrm{Da}$.

Flavanone and flavone aglycones were not found in the analyzed BJ, while their O- and Cglycosides represented the most abundant constituents. The contents of flavonoids, furanocoumarins and cinnamic acid derivatives found in bergamot juice are reported in Table 1. The amount of flavonoids and furanocoumarins were determined using calibration curves obtained with standards, 
while for compounds X1, X2-X3 caffeic and ferulic acid calibration curves were used, respectively. Their amounts were then normalized by the molecular mass ratios. The amount of flavonoids determined in this paper agrees with those reported in literature [17], but they are not in agreement with other studies indicating higher amounts of flavanones [14]. The discrepancy could be ascribed to different factors such as: BJ preparation procedure, BJ obtained from various cultivars, different maturation period or the use of unspecific chromatographic methods not able to separate neoeriocitrin, naringin and hesperidin from their di-oxalate derivatives. Regarding furanocoumarins, we found in BJ bergamottin amounts lower than that reported in literature [17].

Table 1. Content of flavanone-O-glycosides (1-7), flavone-C-glucosides (A-H), flavoneO- neohesperidosides (I-M), cinnamic acid derivatives (X1-3) and furanocoumarins (a-b) in BJ.

\begin{tabular}{|cc|cr|}
\hline Peak & $\mathbf{m g} / \mathbf{L}$ & Peak & \multicolumn{1}{c|}{$\mathbf{~ g / L}$} \\
\hline $\mathbf{1}$ & $9.6 \pm 1.1$ & $\mathbf{F}$ & $2.8 \pm 0.3$ \\
$\mathbf{2}$ & $73.3 \pm 1.6$ & $\mathbf{G}$ & $9.1 \pm 0.7$ \\
$\mathbf{3}$ & $167.5 \pm 1.8$ & $\mathbf{H}$ & $6.0 \pm 0.3$ \\
$\mathbf{4}$ & $20.1 \pm 1.1$ & $\mathbf{I}$ & $46.4 \pm 1.9$ \\
$\mathbf{5}$ & $123.9 \pm 1.7$ & $\mathbf{L}$ & $7.8 \pm 0.4$ \\
$\mathbf{6}$ & $89.3 \pm 2.2$ & $\mathbf{M}$ & $23.1 \pm 1.4$ \\
$\mathbf{7}$ & $190.0 \pm 3.1$ & $\mathbf{a}$ & $9.0 \pm 0.4$ \\
$\mathbf{A}$ & $1.3 \pm 0.1$ & $\mathbf{b}$ & $18.2 \pm 0.5$ \\
$\mathbf{B}$ & $38.6 \pm 2.1$ & $\mathbf{X 1}$ & $1.6 \pm 0.2$ \\
$\mathbf{C}$ & $25.8 \pm 1.3$ & $\mathbf{X 2}$ & $12.6 \pm 0.9$ \\
$\mathbf{D}$ & $2.0 \pm 0.1$ & $\mathbf{X 3}$ & $12.1 \pm 0.8$ \\
$\mathbf{E}$ & $2.1 \pm 0.1$ & & \\
\hline
\end{tabular}

Regarding the validation method, calibration curves were constructed for each standard at five concentration levels and three independent determinations were performed at each concentration. The recovery of the extraction from spiked BJ samples was 97.2 \pm 3.1 . The precision of the method was tested by both intra-day $(n=5)$ and inter-day ( 5 days, $n=5)$ reproducibility, and the coefficient of variation was below $3.3 \%$. Limit of quantization and limit of detection were $2 \mu \mathrm{g} / \mathrm{ml}$ and $1 \mu \mathrm{g} / \mathrm{ml}$, respectively.

\section{Conclusions}

Bergamot juice contains several different compounds and LC-DAD-MS is the best choice for their separation, identification and quantization. Moreover, tandem mass spectrometry with collision induced dissociation allows structural identification, especially when standard compounds are not available. 


\section{Experimental}

\section{Chemicals}

Naringin, narirutin, hesperidin, neohesperidin, neoeriocitrin, isovitexin, rhoifolin, neodiosmin, bergapten and bergamottin were from Extrasynthese (Genay, France). Caffeic and ferulic acid were from Sigma (St. Louis, MO, USA). Methanol, acetonitrile and formic acid were purchased from Merck (Darmstadt, Germany). Water was obtained from a MilliQ apparatus (Millipore, Milford, MA). The bergamot fruits were a gift of the "Consorzio del Bergamotto" (Reggio Calabria, I).

\section{Method validation}

The LC-DAD-MS method was validated for linearity, LOQ, LOD, accuracy, peak purity, precision and repeatability. Lower limit of quantization (LLOQ, $S / N$ ratio of 8 ) and lower limit of detection (LLOD, $S / N$ ratio of 3 ) were determined by serial dilution of standard solutions. Accuracy was evaluated by spiking three BJ sample with four amounts $(2,5,10$ and $20 \mu \mathrm{g} / \mathrm{mL})$ of the mix standard compounds. Peak purity and identity were confirmed by LC-DAD-MS and LC-MS ${ }^{2}$ experiments. Precision (intra- and inter-day) of the assay was verified by analyzing BJ samples 3 times on five consecutive days. Repeatability was confirmed by evaluating standard deviations of the retention times and peaks area.

\section{Sample preparation}

Bergamot fruits $(1 \mathrm{Kg})$ were peeled, squeezed and the obtained juice was mixed and apportioned in $20 \mathrm{~mL}$ containers, which were stored at $-80^{\circ} \mathrm{C}$. Ten $\mathrm{mL}$ were diluted 5-fold in methanol-formic acid $1 \%(90: 10, \mathrm{v} / \mathrm{v})$ and the resulting solution was sonicated for $10 \mathrm{~min}$ and then centrifuged at $1000 \mathrm{xg}$ for $10 \mathrm{~min}$. One aliquot of the supernatant was filtered through a Millipore $0.2 \mu \mathrm{m}$ disk and $5 \mu \mathrm{L}$ were injected into the LC system.

\section{LC-DAD-MS/MS analysis}

The chromatographic system was an Alliance mod. 2695 (Waters, Milford, MA) equipped with a mod. 2996 (Waters) photodiode array detector and a triple quadrupole mass spectrometer mod. Quattro micro (Micromass, Beverly, MA). A $3 \mu \mathrm{m} \mathrm{C}_{18}$ Luna (2) narrow bore column (150 x $2.0 \mathrm{~mm}$, Phenomenex, Torrance, USA) was used for the separation, which was performed by means of a linear gradient elution (eluent A, $0.1 \%$ formic acid; eluent B, acetonitrile) at a flow rate of $250 \mu \mathrm{L} / \mathrm{min}$. The gradient was as follows: from 10 to $25 \% \mathrm{~B}$ in $20 \mathrm{~min}, 25 \% \mathrm{~B}$ for $5 \mathrm{~min}, 25$ to $50 \% \mathrm{~B}$ in $5 \mathrm{~min}, 50$ to $95 \% \mathrm{~B}$ in $5 \mathrm{~min}$ and then $95 \% \mathrm{~B}$ for $15 \mathrm{~min}$. The column was maintained at $30^{\circ} \mathrm{C}$. Chromatographic data were acquired in the 200-450 nm range and were integrated at $282 \mathrm{~nm}$ (flavanones) and $330 \mathrm{~nm}$ (flavones, furanocoumarins and cinnamic acid derivatives). The mass spectrometer operated in positive and negative full-scan mode in the range 100-1000 Da. The capillary voltage was set to 3.0 $\mathrm{kV}$, the cone voltage was $20 \mathrm{~V}$, the source temperature was $130^{\circ} \mathrm{C}$, and the desolvating temperature 
was $350^{\circ} \mathrm{C}$. All data were acquired by Masslink 4.0 software (Micromass) with the Quan-Optimize option for the fragmentation study. Calibration curves were obtained from narirutin, naringin, hesperidin, neohesperidin, rhoifolin, neodiosmin, neoeriocitrin, bergapten, bergamottin, caffeic and ferulic acid stock solutions prepared by dissolving $5 \mathrm{mg}$ of standard powder in $5 \mathrm{~mL}$ methanol. They were measured in the range of $2-20 \mu \mathrm{g} / \mathrm{mL}$.

\section{References}

1. Felgines, C.; Talavera, S.; Texier, O.; Gil-Izquierdo, A.; Lamaison, J.L; Remesy, C. Bioavailability of the flavanone naringenin and its glycosides in rats. Am. J. Physiol. Gastroint. Liver Physiol. 2000, 279, 1148-1154.

2. Matsumoto, H.; Ikoma, Y.; Sugiura, M.; Yano, M.; Hasegawa, Y. Identification and quantification of the conjugated metabolites derived from orally administered hesperidin in rat plasma. J. Agric. Food Chem. 2004, 52, 6653-6659.

3. Hirata, A.; Murakami, Y.; Shoji, M.; Kadoma, Y.; Fujisawa, S. Kinetics of radical-scavenging activity of hesperetin and hesperidin and their inhibitory activity on COX-2 expression. Anticancer Res. 2005, 25, 3367-3374.

4. Shen, S.C.; Ko, C.H.; Tseng, S.W.; Tsai, S.H.; Chen, Y.C. Structurally related antitumor effects of flavanones in vitro and in vivo: involvement of caspase 3 activation, p21 gene expression, and reactive oxygen species production. Toxicol. App. Pharm. 2004, 197, 84-95.

5. Kurowska, E.M.; Manthey, J.A. Hypolipidemic effects and absorption of citrus polymethoxylated flavones in hamsters with diet-induced hyper-cholesterolemia. J. Agric. Food Chem. 2004, 52, 2879-2886.

6. Rotelli, A.E. ; Guardia, T. ; Juarez, AO.; de la Rocha, NE. ; Pelzer, LE. Comparative study of flavonoids in experimental models of inflammation. Pharm. Res. 2003, 48, 601-606.

7. Harris, D.M.; Besselink, E.; Henning, S.M.; Go, V.L.W.; Heber, D. Phytoestrogens induce differential estrogen receptor alpha- or beta-mediated responses in transfected breast cancer cells. Exp. Biol. Med. 2005, 230, 558-568.

8. Edmunds, K.M.; Holloway, A.; Crankshaw, D.J.; Agarwal, S.K.; Foster, W.G. The effects of dietary phytoestrogens on aromatase activity in human endometrial stromal cells. Repr. Nutr. Dev. 2005, 45, 709-720.

9. Jung, U.J.; Kim, H.J.; Lee, J.S.; Lee, M.K.; Kim, H.O.; Park, E.J.; Kim, H.K.; Jeong, T.S.; Choi, M.S. Naringin supplementation lowers plasma lipids and enhances erythrocyte antioxidant enzyme activities in hypercholesterolemic subjects. Clin. Nutr. 2003, 22, 561-568.

10. Kim, H.K.; Jeong, T.S.; Lee, M.K.; Park, Y.B.; Choi, M.S. Lipid-lowering efficacy of hesperetin metabolites in high-cholesterol fed rats. Clin. Chim. Acta 2003, 327, 129-137.

11. Miceli, N.; Mondello, M.R.; Monforte, M.T.; Sdrafkakis, V.; Dugo, P.; Crupi, M.L.; Taviano, M.F.; De Pasquale, R.; Trovato, A. Hypolipidemic effects of Citrus bergamia Risso et Poiteau juice in rats fed a hypercholesterolemic diet. J. Agric. Food Chem. 2007, 55, 10671-10677.

12. Robards, K.; Li, X.; Antolovich, M.; Boyd, S. Characterization of Citrus by chromatographic analysis of flavonoids. J. Sci. Food Agric. 1997, 75, 87-101. 
13. Nogata, Y.; Ohta, H.; Yoza, K.; Berhow, M.; Hasegawa, S. High-performance liquid chromatographic determination of naturally occurring flavonoids in Citrus with a photodiodearray detector. J. Chrom. A 1994, 667, 59-66.

14. Gattuso, G.; Caristi, C.; Gargiulli, C.; Bellocco, E.; Toscano, G.; Lezzi, U. Flavonoid glycosides in Bergamot juice (Citrus bergamia Risso). J. Agric. Food Chem. 2006, 54, 3929-3935.

15. Mandatari, G.; Bennett, R.N.; Bisognano, G.; Saija, A.; Dugo, G.; LO Curto, R.; Faulds, C.B.; Waldron, K. Characterization of flavonoids and pectins from Bergamot (Citrus bergamia Risso) peel, a major by product of essential oil extraction. J. Agric. Food Chem. 2006, 54, 197-203.

16. Ferreres, F.; Silva, B.M.; Andrade, P.B.; Seabra, R.M.; Ferreira, M.A. Approach to the study of C-glycosyl flavones by ion trap HPLC-PAD-ESI/MS/MS: application to seeds of quince (Cydonia oblonga). Phytochem. Anal. 2003, 14, 352-359.

17. Gattuso, G.; Barreca, D.; Caristi, C.; Gargiulli, C.; Lezzi, U. Distribution of Flavonoids and Furocoumarins in Juices from Cultivars of Citrus bergamia Risso. J. Agric. Food Chem. 2007, $55,9921-9927$.

Sample Availability: Available from the authors.

(C) 2008 by the authors; licensee Molecular Diversity Preservation International, Basel, Switzerland. This article is an open-access article distributed under the terms and conditions of the Creative Commons Attribution license (http://creativecommons.org/licenses/by/3.0/). 